\title{
The role of lymph node ultrasound evaluation in melanoma - review of the literature
}

\author{
Loredana Ungureanuํㅜ, Carolina Botar Jid ${ }^{2}$, Elisabeta Candrea ${ }^{1}$, Rodica Cosgarea ${ }^{1}$, \\ Simona Corina Șenilă ${ }^{1}$
}

${ }^{1}$ Department of Dermatology, ${ }^{2}$ Department of Radiology, "Iuliu Hațieganu” University of Medicine and Pharmacy, Cluj-Napoca, Romania

\begin{abstract}
Melanoma is an aggressive tumour, resistant to treatment, derived from melanocytes, with an increasing incidence in the last years in the majority of countries. The most important prognostic factor in the initial stages (I/II) is the presence of metastases at the level of lymph nodes. Ultrasound (US) is a non-invasive method, used in the pre- and post-operative node evaluation due to its high availability, the reduced cost and easy reproducibility. The US accuracy is however dependent on operator expertise. The present article proposes a presentation of the US role in the evaluation of lymph nodes in melanoma patients.

Keywords: melanoma, ultrasound, lymph node, metastasis
\end{abstract}

\section{Introduction}

Melanoma is an aggressive tumour, resistant to treatment, derived from melanocytes, being the neoplasia with the most rapid incidence increase in the Caucasian population in the last 30 years [1]. The prognosis of patients diagnosed with melanoma is excellent in stage I, but it worsens dramatically in advanced stages, which emphasizes the importance of early diagnosis and the importance of correct staging [2]. The most important prognostic factors are the tumour thickness measured by Breslow's index, the presence of ulceration, the mitotic index and the status of regional lymph nodes [2].

Approximately $70 \%$ of melanoma metastases imply the regional lymph nodes, the first draining lymph node being the sentinel lymph node [2]. Consequently, the evaluation of regional lymph nodes status is fundamental in the initial staging and in the establishment of therapy, follow-up and prognosis [3]. The gold standard

Received 18.01.2016 Accepted 10.02.2016

Med Ultrason

2016, Vol. 18, No 2, 224-230

Corresponding author: Loredana Ungureanu

3-6 Clinicilor Street,

400006, Cluj-Napoca, Romania

Phone/Fax: 0040264592394

E-mail: danaszent@yahoo.com in the initial regional lymph node evaluation is the sentinel lymph node biopsy, recommended by international guidlines to be started in the 1B stage [4,5]. This consists of the excision of the sentinel lymph node identified by lymphoscintigraphy and/or the injection of blue dye followed by the conventional histological examination and by immunohistochemistry [6]. This is, however, a surgical staging technique which may have adverse effects, with a morbidity of up to $10 \%$ and a rate of false-negative results between 4 and 20\% [7]. In this context, ultrasound (US) has been proposed as a repeatable non-invasive alternative of evaluating lymph node status.

Although the effective impact of US upon the survival of patients with melanoma is not yet exactly established $[8,9]$, this imaging technique is more and more used in clinical practice, especially in Europe and Australia [10-12] both in the initial evaluation performed before the sentinel lymph node technique and during the follow-up of the patient.

The aim of this paper is to review the current literature regarding the role of lymph node ultrasound in melanoma.

\section{Preoperative evaluation of lymph nodes}

The role of pre-operatory US evaluation of regional lymph nodes in patients newly diagnosed with melanoma 
is disputed [6]. The preoperative lymph node evaluation can be performed in a non-targeted way consisting of the screening of lymph node areas where the draining is supposed to be produced, or in a targeted way by US examination of sentinel lymph nodes identified by lymphoscintigraphy with a radioactive tracer [6].

In one of the first retrospective studies, Kahle et al succeeded in proving the exact localization of the sentinel lymph node in $85 \%$ of patients [13]. Bossi et al demonstrated that US has a sensitivity rate of $89.4 \%$ and a specificity rate of $90.3 \%$ [14]. The first prospective study was realized by Rossi et al, pointing out a specificity of $100 \%$ and a sensitivity of $39 \%$ [15]. In this study, the preoperative US-guided fine-needle aspiration cytology (US-FNAC) correctly detected 12 out of 31 positive lymph node basins [15]. Hocevar et al proved that the ultrasound of regional lymph nodes combined with FNAC allow the correct selection of patients who can directly benefit from a complete regional lymphadenectomy [16]. Subsequent studies could not confirm these results, showing that US is not sufficiently accurate for preoperative staging $[17,18]$. Thus, Sanki et al showed that the sensitivity of US in detecting the metastases in the sentinel lymph node is only $24.3 \%$ comparing with its biopsy [18].

The most recent study of Voit et al showed that US alone can pre-operatory identify up to $71 \%$ of the sentinel lymph node metastases [19]. The confirmation through FNAC leads to a correct detection of $51 \%$ of the involved sentinel lymph node, before the surgical excision [19]. The sensitivity increases in patient subgroups with risk factors for lymph node involvement such as high Breslow thickness (T4 or over $4 \mathrm{~mm}$ ) or the ulceration of the main tumour, leading to a sensitivity of $76 \%$, respectively $61 \%$ [19]. Furthermore, the tumour volume at the level of sentinel lymph node affects the sensitivity of FNAC, resulting in a sensitivity of $17 \%$ in the case of metastases $\leq 0.1 \mathrm{~mm}$ versus $61 \%$ in metastases with a diameter $>1.00 \mathrm{~mm}[19]$. The general sensitivity of $51 \%$ means that $10 \%$ of all patients with melanoma who are subject to the sentinel lymph node biopsy technique, could be spared, and $50 \%$ of patients with positive sentinel lymph node would not have to undertake a biopsy before total lymph node dissection [19].

Regarding survival, Pilko et al proved a smaller tumoral load at the level of the sentinel lymph node and a better survival rate in patients with negative US, compared with the ones with positive US, even in the absence of a positive node aspirate [20]. This observation was subsequently confirmed by Voit et al [19].

Bohelay and al proposed US-guided core needle biopsy (US-CNB) of superficial lymph nodes as an alterna- tive to FNAC for the diagnosis of lymph node metastasis in melanoma, due to high sensitivity, specificity, and positive predictive value (respectively, 97.9, 100, and $100 \%$ ) [21]. US-CNB has similar diagnostic value with US-FNAC but provides relatively large samples of tissue suitable for analyses and it is not associated with adverse events. Due to the small number of cases included (72), larger studies are needed to confirm this results.

Thus, the biopsy of the sentinel lymph node remains the gold standard in the initial node stadialization in melanoma patients, but US combined with fine-needle aspiration cytology allows the selection of patients who can directly undergo a complete regional lymphadenectomy, reducing the number of patients who must undergo the biopsy of the sentinel lymph node [19-23].

\section{The lymph nodes assessment during the patient follow-up}

Despite the continuous increase of melanoma incidence, the mortality caused by it remained relatively constant in the last years, especially due to the efficient treatment in initial stages [1]. Nonetheless, in approximately $50 \%$ of survivors the tumour can relapse, the risk of recurrence being greater in the first years after the diagnosis [24-26]. It was estimated that $20 \%$ of the initial recurrences are local, 50\% appear at the level of regional lymph nodes and $30 \%$ are distant [27-30]. The follow-up of a melanoma patient has the purpose to detect recurrences as soon as possible and with the lowest costs. At the moment, besides the physical examination, the most used imaging methods for the follow-up of melanoma patients are US, computed tomography (CT), positron emission tomography (PET) and PET-CT.

When it comes to the follow-up of patients, it has been proven that a regular US of regional lymph nodes is superior to the clinical examination for detecting metastases, especially the small ones. Blum et al published the first large, prospective study on 1288 patients which proved this superiority [31], and Voit et al showed that the sensitivity of physical examinations in detecting metastases is $25.2 \%$, significantly smaller than the US examinations (99.2\%) [32]. These observations were confirmed by subsequent studies and by a meta-analysis carried out by Bafounta et al [12,33-36]. Moreover, the survival rate of melanoma patients achieved an improvement when using US for a precocious detection of recurrences [36].

A recent meta-analysis performed by Xing et al proved that out of the four imaging methods used for the follow-up, US is superior to CT, PET and PET-CT in detecting lymph node metastases [37]. Taking into consideration the low positive predictive value of CT, PET 
and PET-CT in patients with low risk and the increased costs, US seems to be the chosen imaging method for the lymph node follow-up [37]. On the other hand, PETCT has the highest positive predictive value for the longterm follow-up of distant metastases in patients with high or intermediary risk, or in patients with clinical suspicion [37].

Consequently, the palpation of regional lymph nodes only in the follow-up of patients with melanoma is not enough; performed at regular time intervals, the US is of critical importance, taking into consideration that approximately $2 / 3$ of recurrences appear at the lymph node level, and the early discovery of metastases has been proved to have a positive impact on survival.

\section{Ultrasound diagnostic criteria of nodal metastases in patients with melanoma}

The diagnostic criteria used in the literature for the diagnosis of nodal metastases in the case of melanoma are vague and contradictory, which may explain the contradictions regarding the role of US lymph node assessment [38].

The normal or reactive lymph node has a central area of increased echogenicity, corresponding to the hilar region and a peripheral area of decreased echogenicity corresponding to the cortical parenchyma. Most lymph nodes have a vascular pedicle which achieves a centrally localized perfusion that can disseminate to the periphery in case of inflammatory activation [3].

The first US diagnostic criterion described was the growth of the lymph node due to the presence of malignant cells [38]. Several longitudinal diameters of 3 or 2 $\mathrm{mm}$ were proposed as a cut off, but recent studies showed that the size cannot be used as a single diagnostic criterion [38]. Moreover, Voit et al did not find a correlation between the size of the lymph node and the presence of melanoma metastases [32], and Tregnaghi et al proved that the diagnostic specificity increases when the dimension as a diagnostic criterion is excluded [39]. However, it was demonstrated that the size of the lymph node is important in the US follow-up - the increase of nodes during follow-up is an important sign of malignancy [40].

Another diagnostic criterion derives from the difference in shape: the benign lymph nodes have an oval or elongated shape, whereas the malignant ones have a round or slightly oval shape (fig 1) [38]. The shape of lymph nodes is defined by using the report between their longitudinal and transverse diameters [40]. Nonetheless, the literature does not present a concordance between the longitudinal and transverse diameter suggestive for metastatic nodes, but it suggests values of 1.5 or 2 [38].

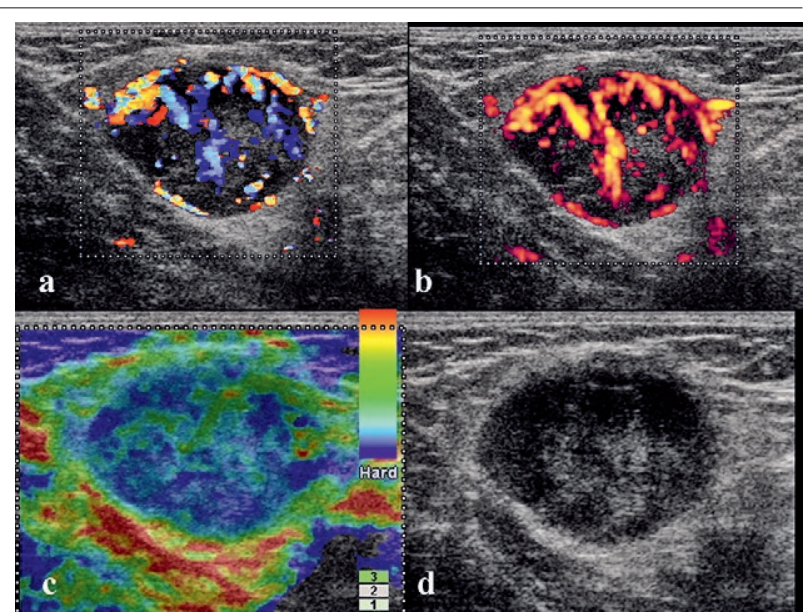

Fig 1. Right inguinal region lymph node, round shape, inhomogeneous, with eccentric hilum, chaotic hypervascularization and stiff, in a case of a 45 year old female with melanoma of the right toe: a) color Doppler; b) power Doppler, c) strain elastography and d) 2D US.

Moreover, the shape can be deceiving taking into consideration that normal or reactive lymph nodes have a round shape in some areas (the parotid or the submandibular area) and in some non-malignant diseases ( such as tuberculosis) the lymph nodes are described as being round [40].

From a morphological standpoint, the absence of echogenic hilum, the presence of a hypoechogenic nodule at the level of the cortex and a well delimited margin were proposed as diagnostic criteria (fig 2) [38]. Normal or reactive lymph nodes have a central echogenic hilum which interrupts the continuity of the cortex and continues with the perinodal fat tissue, in contrast to malignant nodes described as not having a visible hilum [40]. The absence of an echogenic hilum due to replacement or effacement is significantly greater in malignancies, but it can also be present in benign nodes such as reactive ones [40]. As to delimitation, the benign nodes are characterized by a clear delimitation, but this situation can be encountered also in the tumoral nodes [40]. Blurred margins may be observed in acute inflammatory nodes, but also in malignancy, usually indicating extracapsular and extranodal spread and it is associated with a severe prognosis [40].

Structural changes are, most often, encountered in malignant nodes [40]. The presence of hypoechogenic focal cortical nodules, or a focal cortical thickening indicate partial tumour infiltration at the node level and it is a useful sign for identifying metastatic nodes in melanoma patients (fig 3) [40]. Other structural modifications described in malignant nodes are intranodal necrosis, reticulation, calcification, matting or the presence of a pe- 


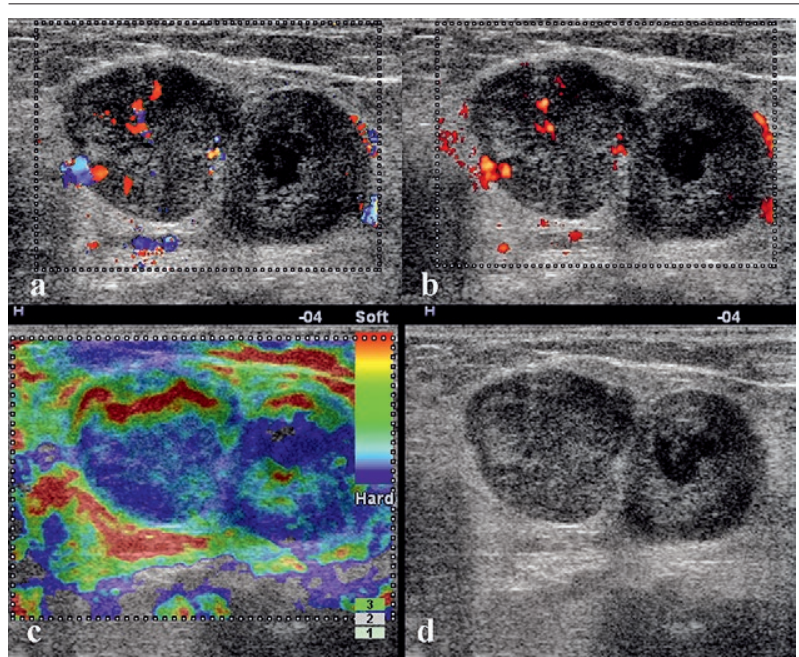

Fig 2. Left inguinal region lymph node, round shape, inhomogeneous, with necrotic areas, eccentric hilum, chaotic hypervascularization, and stiff, in a case of a 57 year old female with melanoma of the left calf: a) color Doppler; b) power Doppler, c) strain elastography and d) 2D US.

ripheral halo or perinodal oedema [40]. All of these were, however, have been described in some benign diseases, like tuberculosis [40].

In the case of colour Doppler examination, the presence of peripheral accessory blood vessels rather tangential than radial towards the hilum, absent or displaced hilar vessels, the existence of avascular asymmetric areas or of an aberrant distribution of central vessels were proposed for the diagnosis of melanomatous metastases [38]. Benign nodes display a single vascular pedicle, entering the hilum while malignant nodes show multiple pedicles, invading the cortex [40].

Thus, melanoma metastases in the superficial lymph nodes appear as oval or rounded nodes without echogenic hilum, with irregular margins and focal cortical hyperplasia [40].

Ulrich et al recently described a set of US morphological models of lymph nodes in melanoma patients; they prospectively tested these criteria, compared them with histopathological changes at the level of excised nodes and correlated them with the tumoral load and the localization of tumour nests (subcapsular $v s$ intraparenchymal) $[41,42]$. The authors were able to describe a morphological model developed in time, from the initial to the tardily involvement [41]. An incipient sign of lymph node alteration, also visible in advanced stages is the emergence of accessory blood vessels in the periphery, named peripheral perfusion (PP) [41,42]. Histopathologically, this change reflects the process through which the nodal tumoral cells determine the formation of a new blood vessel network to help the supply from outside the node

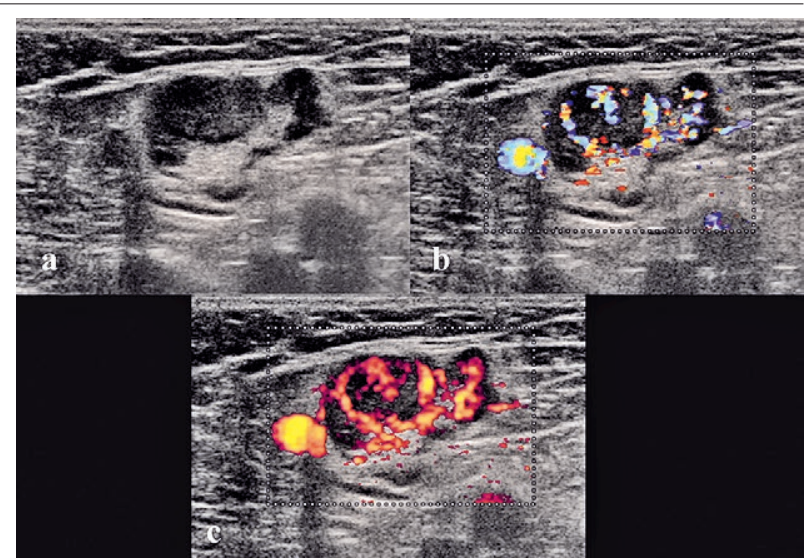

Fig 3. Left inguinal region lymph node oval shape, central hilum, with focal cortical thickening with hypervascularization and stiffness in a case of a 56 year old male with melanoma of the left calf: a) 2D US; b) color Doppler; c) power Doppler.

[41]. The absence of the echogenic hilum or the loss of central echoes (LCE) is a sign which appears after or simultaneously with PP [41]. Initially, central echoes are only displaced, but after the total lymph node involvement the central echoes are completely lost [41]. This leads to the change of the nodal shape and to the final appearance of the balloon-shaped (BS) node, equivalent with the massive involvement of the whole node [40]. Thus, the authors consider that PP is an incipient sign, sufficient for FNAC, although the latter will not always prove the presence of nodal metastases which can be missed due to its small size [41]. The authors also consider that LCE and BS are unmistakable signs of advanced involvement in $96 \%$ of cases [41].

Moreover, Voit et al have managed to correlate the overall survival (OS) with US aspect [43]. Thus, after 5 years, OS was $93 \%$ in patients without PP and BS, $87 \%$ in patients with PP only, and 56\% in patients with BS nodes, with or without LCE [41]. In the three categories mentioned above, the disease-free survival was of 74, 60 and $26 \%$ respectively [41].

\section{New US techniques used in lymph node assessment}

In the last years sonoelastography (SE), was introduced for the assessment of superficial lymph nodes, technique which describes the relative stiffness of tissue [44]. The hypothesis is that cells in solid tumours differ in consistency from adjacent normal tissue [44]. In SE, inflammatory or reactive nodes have the same appearance as the soft tissue of the neck and are hardly visible as distinct entities. Benign nodes display mostly colours as yellow, green and turquoise; the red colour is absent and blue is rarely and inconstantly present. On the col- 
our scale, the cortex appears harder than the medulla [44] while malignant nodes stand out from the surrounding tissue due to their increased stiffness. Blue areas, representing hard, rigid tissue, occupy more than $45 \%$ of the lymph node; red, yellow and green are not encountered in malignant areas. The cortex is stiffer than the medulla or the node is uniformly hard [44]. For a better SE differentiation between benign and malignant nodes, grading of the appearance on a score system (elasticity score - ES) or calculation of the relative stiffness or strain ratio (SR) can be done. Hardness on more than $50 \%$ of the node surface or SR > 1.5 are fair to good indicators of malignancy [44].

Hinz et al conducted a study to investigate the value of real-time SE for the differentiation of benign and metastatic peripheral lymph nodes in melanoma patients by comparing this technique with conventional B-mode sonography combined with power Doppler sonography [45]. Significant differences in elastographic patterns were found between metastatic and nonmetastatic lymph nodes. An elastography pattern $\geq 3$ (considering the proportion of stiff areas in a score from 1 to 5) was identified as an independent significant factor, predicting a metastatic involvement. The combination of SE with conventional B-mode sonography has the potential to further improve the differentiation between benign and metastatic lymph nodes in melanoma patients [45].

Another study was conducted by Ogata et al in order to document the accuracy of real-time SE for the differential diagnosis of reactive versus metastatic lymph nodes in cutaneous melanoma patients [46]. The authors used a scoring system which was simplified and modified from the 5-point color scoring of Alam et al [47]. The percentage of blue area was measured subjectively. They concluded that SE can enhance the diagnostic accuracy of ultrasound for differentiating between reactive and malignant nodes in melanoma. The optimum ES cut off value for reactive versus metastatic lymph nodes is 4 , equivalent to large blue areas in the lymph node, the total blue area covering more than $50 \%$ of the node [46]. Thus, routine preoperative US combining B-mode images and $\mathrm{SE}$ in melanoma patients may be practical to eliminate the need for sentinel lymph node biopsy [46].

Another US-technique described recently in the melanoma lymph nodes assessment is the contrast-enhanced ultrasound (CEUS) imaging to detect lymphatic channels and sentinel lymph nodes after interstitial injections of microbubble-based contrast agents (termed lymphosonography). This technique was first described in a melanoma tumor animal model by Liu et al [48]. The authors showed that secondary lymph nodes identified with CEUS were significantly more likely to contain metastases than those removed by radical dissection. The potential benefit of this technique is the development of a minimally invasive diagnostic imaging method that can be used to identify lymphatic drainage from tumors to the sentinel lymph node and onward to secondary lymph nodes [48]. This technique could be performed both preoperatively as well as intraoperatively [48].

Rubaltelli at al were the first to conduct a study regarding the role of CEUS in melanoma patients [49]. They concluded that CEUS increases the diagnostic accuracy of US in the differential diagnosis of benign and malignant lymph nodes but it also allows the avoiding of unnecesary invasive operations such as US-FNAC [49]. Moreover, CEUS may guide FNAC in the case of focal cortical thickening on the basis of hypoperfusion, with a reduction in the number of false negatives and much earlier detection of nodal metastatic foci [49].

Freesmeyer et al described recently a new hybrid freehand SPECT/ultrasound (fh-SPECT/US) imaging concept, with regard to sentinel lymph node imaging, in patients with breast cancer and malignant melanoma [50]. Following standard sentinel lymph node scintigraphy, fh-SPECT was performed, a handheld-gamma camera-based method to visualize activity distribution within a region of interest as a cross-sectional data set. These data were transferred to an US device and sensor-navigated US was performed combining fh-SPECT data with US images, displaying superimposed images. Quality of fh-SPECT and co-registration accuracy was assigned to one of four categories and occurrence of artefacts was assessed [50]. The fusion imaging concept proved to be feasible and technically successful. However, significant technical limitations were shown in fh-SPECT quality and fusion precision. The authors concluded that subject to the technical optimisation of SPECT quality and coregistration, a meaningful contribution to the preoperative planning of lymph node therapy is imaginable. Thus, fundamentally a preoperative histological examination by fh-SPECT/US-guided biopsy is possible [50].

\section{Conclusions}

The US techniques have an important role nowadays in the stadialization, treatment and follow-up of melanoma patients as it is a simple and repeatable technique, which needs however, experience. The US evaluation before the sentinel lymph node biopsy is a developing domain, but combined with US-FNAC it can help in the selection of patients who can undergo a lymph node dissection, protecting them from a useless operation. The new developing US techniques seem to be promising in the preoperative setting. During follow-up, US rep- 
resents the most accurate imaging method for detecting nodal metastases, superior to CT, PET and PET-CT. The new SE technique seems to enhance the diagnostic accuracy of US in differentiating benign from malignant lymph nodes in melanoma patients, but further validation in larger studies is needed.

\section{Conflict of interest: none}

\section{References}

1. MacKie RM, Hauschild A, Eggermont AM. Epidemiology of invasive cutaneous melanoma. Ann Oncol 2009; 20(Suppl 6): vi1-vi7.

2. Balch CM, Gershenwald JE, Soong SJ, et al. Final version of 2009 AJCC melanoma staging and classification. J Clin Oncol 2009; 27: 6199-6206.

3. Schäfer-Hesterberg G, Schoengen A, Sterry W, Voit C. Use of ultrasound to early identify, diagnose and localize metastases in melanoma patients. Expert Rev Anticancer Ther 2007; 7: 1707-1716.

4. Marsden JR, Newton-Bishop JA, Burrows L, et al. Revised UK guidelines for the management of cutaneous melanoma 2010. J Plast Reconstr Aesthet Surg 2010; 63: 1401-1419,

5. Garbe C, Peris K, Hauschild A, et al. Diagnosis and treatment of melanoma: European consensus-based interdisciplinary guideline. Eur J Cancer 2010; 46: 270-283.

6. Thompson JF, Haydu LE, Sanki A, Uren R. Ultrasound assessment of lymph nodes in the management of early stage melanoma. J Surg Oncol 2011; 104: 354-360.

7. van Akkooi AC, Voit CA, Verhoef C, Eggermont AM. New developments in sentinel node staging in melanoma: controversies and alternatives. Curr Opin Oncol 2010; 22: 169-177.

8. Machet L, Nemeth-Normand F, Giraudeau B, et al. Is ultrasound lymph node examination superior to clinical examination in melanoma follow-up? A monocentre cohort study of 373 patients. Br J Dermatol 2005; 152: 66-70.

9. Machet L, Perrinaud A, Giraudeau B. Routine ultrasonography in melanoma follow-up. Lancet Oncol 2005; 6: 2.

10. Catalano O, Siani A. Cutaneous melanoma: role of ultrasound in the assessment of locoregional spread. Curr Probl Diagn Radiol 2010; 39: 30-36.

11. Voit C, Schoengen A, Schwürzer-Voit M, et al. The role of ultrasound in detection and management of regional disease in melanoma patients. Semin Oncol 2002; 29: 353-360.

12. Garbe C, Paul A, Kohler-Späth H, et al. Prospective evaluation of a follow-up schedule in cutaneous melanoma patients: recommendations for an effective follow-up strategy. J Clin Oncol 2003; 21: 520-529.

13. Kahle B, Hoffend J, Wacker J, Hartschuh W. Preoperative ultrasonographic identification of the sentinel lymph node in patients with malignant melanoma. Cancer 2003; 97: 1947-1954.

14. Bossi MC, Sanvito S, Lovati E, De Fiori E, Testori A, Bellomi M. Role of high resolution color-Doppler US of the sentinel node in patients with stage I melanoma. Radiol Med 2001; 102: 357-362.

15. Rossi CR, Mocellin S, Scagnet B, et al. The role of preoperative ultrasound scan in detecting lymph node metastasis before sentinel node biopsy in melanoma patients. J Surg Oncol 2003; 83: 80-84.

16. Hocevar M, Bracko M, Pogacnik A, et al. The role of preoperative ultrasonography in reducing the number of sentinel lymph node procedures in melanoma. Melanoma Res 2004; 14: 533-536.

17. van Rijk MC, Teertstra HJ, Peterse JL, et al. Ultrasonography and fine-needle aspiration cytology in the preoperative evaluation of melanoma patients eligible for sentinel node biopsy. Ann Surg Oncol 2006; 13: 1511-1516.

18. Sanki A, Uren RF, Moncrieff M, et al. Targeted high-resolution ultrasound is not an effective substitute for sentinel lymph node biopsy in patients with primary cutaneous melanoma. J Clin Oncol 2009; 27: 5614-5619.

19. Voit CA, Gooskens SL, Siegel P, et al. Ultrasound-guided fine-needle aspiration cytology as an addendum to sentinel lymph node biopsy can perfect the staging strategy in melanoma patients. Eur J Cancer 2014; 50: 2280-2288.

20. Pilko G, Zgajnar JS, Music M, Hocevar M. Lower tumour burden and better overall survival in melanoma patients with regional lymph node metastases and negative preoperative ultrasound. Radiol Oncol 2012; 46: 60-68.

21. Bohelay G, Battistella M, Pagès C, et al. Ultrasound-guided core needle biopsy of superficial lymph nodes: an alternative to fine-needle aspiration cytology for the diagnosis of lymph node metastasis in cutaneous melanoma. Melanoma Res 2015; 25: 519-527.

22. Schafer-Hesterberg G, Schoengen A, Sterry W, Voit C. Use of ultrasound to early identify, diagnose and localize metastases in melanoma patients. Expert Rev Anticancer Ther 2007; 7: 1707-1716.

23. Voit CA, van Akkooi AC, Schaefer-Hesterberg G, Schoengen A, Sterry W, Eggermont AM. Role of ultrasound (US) and US-guided fine needle aspiration cytology (US-FNAC) prior to sentinel lymph node biopsy (SLNB) in 500 melanoma patients: reduction of need for SLND by high USFNAC SN positive identification rate. J Clin Oncol (Meeting Abstracts) 2007; $2518 \mathrm{~S}: 8512$.

24. McEwan L, Smith JG, Matthews JP. Late recurrence of localized cutaneous melanoma: its influence on follow-up policy. Plast Reconstr Surg 1990; 86: 527-534.

25. Slingluff CL Jr, Dodge RK, Stanley WE, Seigler HF. The annual risk of melanoma progression. Implications for the concept of cure. Cancer 1992; 70: 1917-1927.

26. Romero JB, Stefanato CM, Kopf AW, Bart RS. Follow-up recommendations for patients with stage I malignant melanoma. J Dermatol Surg Oncol 1994; 20: 175-178.

27. Benvenuto-Andrade C, Oseitutu A, Agero AL, Marghoob AA. Cutaneous melanoma: surveillance of patients for recurrence and new primary melanomas. Dermatol Ther 2005; 18: 423-435.

28. Soong SJ, Harrison RA, McCarthy WH, Urist MM, Balch $\mathrm{CM}$. Factors affecting survival following local, regional, or 
distant recurrence from localized melanoma. J Surg Oncol 1998; 67: 228-233

29. Meier F, Will S, Ellwanger U, et al. Metastatic pathways and time courses in the orderly progression of cutaneous melanoma. Br J Dermatol 2002; 147: 62-70.

30. Dicker TJ, Kavanagh GM, Herd RM, et al. A rational approach to melanoma follow-up in patients with primary cutaneous melanoma. Scottish Melanoma Group. Br J Dermatol 1999; 140: 249-254.

31. Blum A, Schlagenhauff B, Stroebel W, Breuninger H, Rassner G, Garbe C. Ultrasound examination of regional lymph nodes significantly improves early detection of locoregional metastases during the follow-up of patients with cutaneous melanoma: results of a prospective study of 1288 patients. Cancer 2000; 88: 2534 -2539.

32. Voit C, Mayer T, Kron M, et al. Efficacy of ultrasound Bscan compared with physical examination in follow-up of melanoma patients. Cancer 2001; 91: 2409-2416.

33. Schmid-Wendtner MH, Paerschke G, Baumert J, Plewig $\mathrm{G}$, Volkenandt M. Value of ultrasonography compared with physical examination for the detection of locoregional metastases in patients with cutaneous melanoma. Melanoma Res 2003; 13: 183-188.

34. Hofmann U, Szedlak M, Rittgen W, Jung EG, Schadendorf D. Primary staging and follow-up in melanoma patients monocenter evaluation of methods, costs and patient survival. Br J Cancer 2002; 87: 151-157.

35. Leiter U, Marghoob AA, Lasithiotakis K, et al. Costs of the detection of metastases and follow-up examinations in cutaneous melanoma. Melanoma Res 2009; 19: 50-57.

36. Bafounta ML, Beauchet A, Chagnon S, Saiag P. U1trasonography or palpation for detection of melanoma nodal invasion: a meta-analysis. Lancet Oncol 2004; 5: 673-680.

37. Xing Y, Bronstein Y, Ross MI, et al. Contemporary diagnostic imaging modalities for the staging and surveillance of melanoma patients: a meta-analysis. J Natl Cancer Inst 2011; 103: 129-142.

38. Catalano O. Critical analysis of the ultrasonographic criteria for diagnosing lymph node metastasis in patients with cutaneous melanoma: a systematic review. J Ultrasound Med 2011; 30: 547-560.

39. Tregnaghi A, De Candia A, Calderone M, et al. Ultrasonographic evaluationof superficial lymph node metastases in melanoma. Eur J Radiol 1997; 24: 216-221.
40. Dudea SM, Lenghel M, Botar-Jid C, Vasilescu D, Duma M. Ultrasonography of superficial lymph nodes: benign vs. malignant. Med Ultrason 2012; 14: 294-306.

41. Ulrich J, van Akkooi AJ, Eggermont AM, Voit C. New developments in melanoma: utility of ultrasound imaging (initial staging, follow-up and pre-SNLB). Expert Rev Anticancer Ther 2011; 11: 1693-1701.

42. Ulrich J, van Akkooi AC, Eggermont AM, Voit CA. Sonographic criteria for diagnosing sentinel node metastases in melanoma patients. Ultraschall Med 2015; 36: 149-153.

43. Voit CA, van Akkooi AC, Schafer-Hesterberg G, et al. Rotterdam Criteria for sentinel node (SN) tumor burden and the accuracy of ultrasound (US)-guided fine-needle aspiration cytology (FNAC): can US-guided FNAC replace SN staging in patients with melanoma? J Clin Oncol 2009; 27: 4994-5000.

44. Dudea SM, Botar-Jid C, Dumitriu D, Vasilescu D, Manole $\mathrm{S}$, Lenghel ML. Differentiating benign from malignant superficial lymph nodes with sonoelastography. Med Ultrason 2013; 15: 132-139.

45. Hinz T, Hoeller T, Wenzel J, Bieber T, Schmid-Wendtner $\mathrm{MH}$. Real-time tissue elastography as promising diagnostic tool for diagnosis of lymph node metastases in patients with malignant melanoma: a prospective single-center experience. Dermatology 2013; 226: 81-90.

46. Ogata D, Uematsu T, Yoshikawa S, Kiyohara Y. Accuracy of real-time ultrasound in the differential diagnosis of lymph nodes in cutaneous malignant melanoma (CMM): a pilot study. Int J Clin Oncol 2014; 19: 716-721.

47. Alam F, Naito K, Horiguchi J, Fukuda H, Tachikake T, Ito $\mathrm{K}$. Accuracy of sonographic elastography in the differential diagnosis of enlarged cervical lymph nodes: comparison with conventional B-mode sonography. AJR Am J Roentgenol 2008; 191: 604-610.

48. Liu JB, Merton DA, Berger AC, et al. Contrast-enhanced sonography for detection of secondary lymph nodes in a melanoma tumor animal model. J Ultrasound Med 2014; 33: 939-947.

49. Rubaltelli L, Beltrame V, Scagliori E, et al. Potential use of contrast-enhanced ultrasound (CEUS) in the detection of metastatic superficial lymph nodes in melanoma patients. Ultraschall Med 2014; 35: 67-71.

50. Freesmeyer M, Winkens T, Opfermann T, Elsner P, Runnebaum I, Darr A. Real-time ultrasound and freehand-SPECT. Experiences with sentinel lymph node mapping. Nuklearmedizin 2014; 53: 259-264. 